\title{
Coulisses
}

Revue de théâtre

1 | Printemps 1990

Varia

\section{Freud et le théâtre}

\section{Yves Thoret}

\section{(2) OpenEdition}

\section{Journals}

Édition électronique

URL : http://journals.openedition.org/coulisses/1564

DOI : $10.4000 /$ coulisses.1564

ISSN : 2546-9460

\section{Éditeur}

Presses universitaires de Franche-Comté

\section{Édition imprimée}

Date de publication : 1 avril 1990

Pagination : 55-66

ISSN : 1150-594X

\section{Référence électronique}

Yves Thoret, «Freud et le théâtre », Coulisses [En ligne], 1 | Printemps 1990, mis en ligne le 04 juillet

2017, consulté le 21 octobre 2019. URL : http://journals.openedition.org/coulisses/1564 ; DOI :

10.4000/coulisses.1564

Ce document a été généré automatiquement le 21 octobre 2019

Coulisses 


\title{
Freud et le théâtre
}

\author{
Yves Thoret
}

\section{NOTE DE L'ÉDITEUR}

Cet article est en quelque sorte la théorie de l'atelier que l'auteur animera avec André Bénichou du Théâtre populaire jurassien « Psychanalyse et direction d'acteurs » lors des Rencontres internationales « Théâtre et université » (19 avril 1990).

\section{NOTE DE L'AUTEUR}

Travail effectué au Centre de recherches et d'études freudiennes (CREF), université de Paris X-Nanterre.

\section{Le rêve comme spectacle}

1 Nous connaissons cette analogie fondamentale que Freud établit entre le théâtre et cette "autre scène où le rêve se meut, cette autre scène, cet autre plateau scénique (Schauplatz), selon l'expression de Fechner, où se joue l'action du rêve et qui est différente de celle de la vie éveillée ${ }^{1} . .$. ». C'est grâce à cette formule de Fechner que Freud a pu découvrir le secret de l'interprétation des rêves.

2 Je souhaite attirer votre attention sur certains mécanismes en jeu dans la psychologie normale et pathologique que nous comprenons mieux en analysant leur analogie avec le spectacle de théâtre.

3 Freud salue la précision avec laquelle F.W. Hildebrandt, en 1875, décrivait les caractères de ce qu'il appelait «la forme dramatique » de représentation adoptée par le rêve ; le rêve exerce une opération de compression portant sur les procédés de raisonnement les plus compliqués en une fraction de temps la plus brève possible. Ainsi conclut 
Hildebrandt, «le rêve a dramatisé les paroles de l'Apôtre : Celui-là qui hait son frère, celui-là est un assassin ${ }^{2} »$.

4 Freud reprendra l'équivalence rappelée par Oscar Panizza dans Le Concile d'amour: «Penser et faire sont une même chose ${ }^{3}$ " pour décrire un mécanisme spécifique du travail du rêve, la dramatisation. Il définit ce mécanisme comme la «transformation d'une idée en situation », en scène ${ }^{4}$.

Dès qu'il a décrit ce mécanisme de dramatisation, Freud lui reconnaît un pouvoir actif. La scène choisie, remémorée ou imaginée par l'action de ce mécanisme va constituer elle-même un point de cristallisation qui va attirer à lui les matériaux des pensées du rêve, les redistribuer, en modifier l'emplacement, l'éclairage, l'apparence, le relief. Ce noyau de cristallisation est le pivot, le centre, l'argument autour duquel vont se greffer de multiples révisions, interpolations, ajouts, modifications qui transforment partiellement la scène originaire pour lui donner de l'ampleur et la richesse d'un spectacle, c'est le travail de la scène.

6 Dès lors, on peut distinguer deux aspects complémentaires de ce mécanisme, la mimésis et le jeu.

7 D'une part, la dramatisation dans le rêve fait revivre le plus fidèlement possible un moment du passé, ou un épisode important de notre vie, avec certains détails très concrets de la situation. On retrouve ici la notion de mimésis qui est à la base des arts du spectacle. Le rêve est la copie d'un original, d'une scène ancienne oubliée ou enfouie dans le coffre de nos souvenirs d'enfance.

D'autre part, le travail du rêve et le travail de la scène introduisent une dimension de jeu qui est une autre composante fondamentale du théâtre, la transposition, le jeu théâtral. Le rêve transforme la scène dont il présente la reproduction, il transpose ses éléments, il travestit certains aspects, joue avec le canevas de la scène rappelée. Freud insiste sur le fait que, pour mériter le nom de rêve, il faut que s'opère un travail par lequel «les éléments du rêve sont condensés, leurs accents psychiques déplacés et l'ensemble du matériel préconscient traduit en images visuelles ou dramatisé ${ }^{5} »-$ C'est $^{\prime}$ cette dernière opération qui domine selon lui le processus du rêve.

9 Bien sûr la dramatisation comporte un premier niveau de mise en image mais elle exerce aussi un travail de mise en scène. Le terme "Dartelbarkeit» qu'on traduit généralement par "figurabilité », veut dire plus qu'une simple mise en image ou représentation figurative ; Lacan proposait de le traduire par « égard aux moyens de la mise en scène ${ }^{6}$ » et Didier Anzieu choisit le terme " représentabilité ${ }^{7}$ » aptitude à être joué, à être représenté.

10 Ainsi, la dramatisation opère un mouvement de régression associant la mise en image à la mise en scène d'une pensée de désir.

Une pensée, en règle une pensée de désir est objectivée dans le rêve, représentée comme une scène (Als Szene dargestellt) ou, à ce qu'il nous semble, vécue (erlebt).

11 Voilà ce qui donne à la dramatisation son pouvoir de reviviscence. Le théâtre apparaît ici comme la référence qui permet de comprendre la régression formelle, temporelle et topique du rêve, «la représentation retourne à l'image sensorielle d'où elle est sortie un jour ${ }^{9} »$ - Elle apparaît vécue au présent, actualisée ; le désir y est mis en scène sous la forme de sa réalisation objective, accomplie, triomphante. 
12 On retrouve la même référence au théâtre quand Freud cherche à décrire les manifestations de l'hystérie. La phase des attitudes passionnelles peut se comprendre, écrit-il, comme « la reviviscence hallucinatoire d'une scène ${ }^{10}$ ».

De même, il utilise une métaphore théâtrale en 1912 pour décrire comment une idée inconsciente peut trouver une expression dans le symptôme sans pour autant devenir manifeste à la conscience du sujet; une patiente hystérique présentant des vomissements liés à l'idée inconsciente d'être enceinte peut observer son accès avec «l'attitude détachée d'un spectateur », restant inconsciente de l'incident dont « elle joue la reproduction dramatique ${ }^{11} »$.

14 Tout ce travail par lequel le souvenir, croche par le désir inconscient, cherche à reprendre vie sur la scène substitutive du rêve, entre en rapport avec ce que Spitta appelle la vie affective, le cœur du sujet, ses sentiments les plus intimes, das Gemutsleben $^{12}$.

15 C'est dans cette zone dont nous refusons souvent de reconnaître l'existence que, comme l'écrivait, dès 1875, Hildebrandt, « nous parlons le langage de la vérité, quoique inconsciemment ${ }^{13} »$. L'auteur français Delboeuf avait décrit en ces termes le rêveur soumis à ses pulsions : " Le songeur est un acteur qui joue à volonté les fous et les sages, les bourreaux et les victimes, les nains et les géants, les démons et les anges ${ }^{14}$. " Si nous revenons à la belle description de la forme dramatique du rêve selon Hildebrandt, nous voyons évoqués « les modes les plus compliqués du raisonnement ».

Dans l'interprétation des rêves, Freud illustre la dramatisation par un exemple clinique: la contagion hystérique ${ }^{15}$. Une patiente dans une chambre rassemblant plusieurs malades présente un accès de tremblement. Le lendemain sa voisine sera porteuse du même symptôme ; si on parle de " contagion hystérique », il faut se garder de réduire cela à une simple imitation. La voisine ne fait pas simplement « comme » la première patiente. Elle opère tout un travail complexe de raisonnement inconscient dont elle déduit que les causes profondes qui ont bouleversé sa compagne pourraient éventuellement, tout aussi bien, la bouleverser elle-même et elle se trouve ainsi conduite non seulement à « imiter » sa voisine mais « à s'identifier » à elle, à passer du « comme si » de l'imitation superficielle, au «tout comme si » de la résonnance, ou de l'écho entre deux raisons qui lui ont fait sentir profondément le drame ressenti par l'autre patiente, et l'ont amenée par cet écho affectif, cette communauté de sentiments, a rejouer à retrouver de l'intérieur tout le cheminement rhétorique, démonstratif du symptôme.

17 Sans une comparaison passionnée avec le travail d'acteur, Freud n'aurait pas pu, me semble-t-il, décrire avec tant d'à-propos cette connivence, cette reconnaissance des mêmes ressorts cachés de l'émotion, du sentiment, de l'inquiétude, du désir de partager une expérience intense avec celui qui la vit devant nous.

18 De la même manière, Freud décrit en $1917^{16}$ comment une image sensorielle très investie par un fantasme peut s'exprimer de manière hallucinatoire sur l'écran de la perception, non pas en provenance du monde extérieur comme dans la perception sensorielle habituelle, mais en provenance de l'intérieur du sujet, frappant ainsi la face interne de l'écran de la perception et donnant au sujet l'illusion d'une scène qui " crève l'écran » du système perception-conscience et apparaît "reconnue comme une meilleure réalité ». 


\section{2. «Personnages psychopathologiques à la scène »}

19 Ce petit article de sept pages est consacré entièrement au théâtre ${ }^{17}$. Il fut écrit par Freud en 1905 et publié après sa mort par son ami le musicologue Max Graf, à qui il avait confié le manuscrit.

En lisant mon premier chapitre sur le rêve vous avez pu penser que je donnais une trop grande place au travail d'identification, aux attitudes de type hystérique, au souci d'entrer dans la peau du personnage, en négligeant le mécanisme d'illusion, particulièrement complexe au théâtre.

L'analyse de cet article de 1905 que j'appellerai «personnages... » va me permettre de nuancer mon propos.

Cet article est centré sur un mécanisme principal, la catharsis $^{18}$. Freud étudie cette notion décrite par Aristote dans sa définition célèbre de la tragédie : celle-ci, « suscitant pitié et crainte, opère la catharsis propre à pareilles émotions ». On peut rappeler l'intérêt des travaux de deux philologues contemporains de Freud, le français Henri Weil et l'oncle de Martha, Jakob Bernays (professeur de philologie classique à l'Université de Bonn) ainsi que les thèses récentes de Hans Robert Jauss et de l'Ecole de Constance) pour distinguer dans la catharsis trois aspects :

- une dimension psychologique, l'émotion cathartique,

- une dimension esthétique, le plaisir cathartique,

- et enfin, une dimension rhétorique, la communication cathartique.

a) L'émotion cathartique est son aspect le plus connu; un sujet dans un groupe ou au cours d'un entretien fond en larmes et exprime brutalement un souvenir pénible ou une représentation très personnelle.

Pour Aristote, fils de médecin, ce mécanisme désignait une bonne purge. Freud ${ }^{19}$ insiste sur cet aspect économique de vidange, de jaillissement (Eroffnung), de décharge où les affects personnels se donnent libre-cours (Austoben).

b) Le second aspect est le plaisir cathartique, son aspect esthétique, ce qui se libère si violemment chez le spectateur, ce sont les sources du plaisir et de la jouissance.

Or ce plaisir nous envahit tantôt quand nous nous identifions au héros dans son triomphe, sa puissance, son panache, son pouvoir de tout soumettre à son désir, mais tantôt aussi, de manière paradoxale, quand le personnage plonge dans l'ombre du tragique et que nous frissonnons de compassion (Mitleiden) et de crainte.

Henri Weil « ne peut s'empêcher de penser que la catharsis dont parle Aristote doit être une espèce de plaisir, et que le philosophe désigne par ce terme cette jouissance si particulière que nous procurent les émotions tragiques »; le plaisir du spectateur d'une tragédie est « la satisfaction du besoin que nous avons de telles émotions ${ }^{20}$ ».

Le spectateur admire la grandeur du héros tragique mais ressent aussi sa puissante satisfaction masochique dans la défaite : Freud note avec un certain mépris comment le spectateur se laisse en quelque sorte "récupérer" par un plaisir momentané et mesquin de caractère masochique.

c) On peut décrire enfin un troisième aspect de la catharsis, une dimension rhétorique, une forme de communication, propice à la persuasion, à l'acquisition de convictions nouvelles et originales, à ce qu'Aristote appelle la «reconnaissance» (anagnorisis, 
Anerkennung) qui est un "passage de l'ignorance à la connaissance", la découverte d'une problématique, l'ouverture à une signification, la rencontre d'une identitée ${ }^{1}$.

Freud a magistralement illustré cela dans son commentaire d'ÆEdipe roi en 1897: "Chaque spectateur fut un jour, en germe, en imagination, un ÆEdipe et s'épouvante devant la réalisation de son rêve transposé dans la réalité ${ }^{22}$."

Mais, la catharsis n'apparait, Freud insiste sur ce point en 1905, que si le spectateur de théâtre réalise une double opération contradictoire préalable, constituant l'illusion scénique,

- d'une part, il s'identifie au héros, c'est la motion d'adhésion, de croyance, d'enchantement ;

- d'autre part, il se rappelle en permanence que tout cela n'est qu'un jeu, que c'est un autre qui agit et souffre sur la scène, que cet autre est joué par un acteur, que ce n'est qu'un spectacle, qu'un simulacre, qu'une représentation s'insérant dans l'espace-temps protégé par la convention théâtrale.

Ce double mouvement, cette association paradoxale d'une motion de croyance et d'une motion de démenti, de méta-représentation critique, Freud désigne l'ensemble de ce mécanisme du nom d'illusion.

Point n'est donc besoin d'avoir recours aux mécanismes bien connus de dénégation ou de déni, le terme d'illusion est celui choisi par Freud pour décrire cette opération paradoxale. Il en fait un préalable à toute expérience de jouissance et de catharsis au théâtre ; il écrit :

Aussi la jouissance du spectateur pré-suppose-t-elle l'illusion [Illusion en allemand], c'est-à-dire l'adoucissement de la souffrance par l'assurance [Sicherheit] que, premièrement c'est un autre qui agit et souffre là-bas sur la scène, et que deuxièmement ce n'est finalement qu'un jeu d'où il ne peut découler aucun dommage pour sa sécurité personnelle ${ }^{23}$.

Dès lors, ce mécanisme d'illusion décrit ainsi par Freud, nous paraît correspondre parfaitement à une des définitions qu'en donne le Dictionnaire Littré de la langue française et que j'ai proposé d'appeler « illusion paradoxale ${ }^{24}$ ».

Ainsi peut-on comprendre tout le jeu qui s'opère au théâtre, cette dimension ludique présente dans l'étymologie du mot «illusion» («inlusum » de ludere: jouer, jouer dans) et qui relie le spectateur à l'acteur par le regard ${ }^{25}$. L'action spécifique du spectateur est de « participer par le regard au jeu théâtral » et Freud va écrire «Schau-spiel » avec un tiret pour bien insister sur le rapport spécifique du regard du spectateur à un jeu théâtral, à la métamorphose qui transforme l'homme ordinaire en héros, mais surtout qui transforme un texte en une action jouée, en une scène.

Dans « Personnages psychopathologiques à la scène », on peut distinguer deux parties :

- la première, que nous venons d'analyser, où Freud démontre que la catharsis peut procurer à la fois soulagement et excitation par la libération de motions pulsionnelles.

- dans une deuxième partie, annonçant la deuxième topique, Freud s'aventure dans les rapports touffus et confus du théâtre avec la névrose, en insistant sur la notion de résistance.

Après une citation des classifications classiques de Hegel et Lessing distinguant dans la création littéraire, poésie lyrique, épopée et drame, il rappelle la règle d'or du dramaturge : ne pas faire souffrir le public et compenser toute expérience pénible par un plaisir parallèle, portant sur les qualités formelles et esthétiques du spectacle. Ce plaisir formel peut provenir de la beauté du décor, de l'élégance des silhouettes et des costumes, de la musicalité du texte, de la fraîcheur du jeu, de la finesse de la mise en 
scène, de la troublante poésie du spectacle dans la gamme variée de ses messages polysensoriels. Toutes ces sources de plaisir constituent la prime d'attirance, le plaisir préliminaire, le bonus du plaisir qui «tamponne » ou neutralise l'angoisse ressentie et ainsi évite la mise en jeu du refoulement.

Ce qui apparaît ici comme un élément nouveau, en contrepoint de la découverte du refoulé, c'est la notion de résistance. Cette résistance opère, elle aussi, comme le refoulement, a un niveau inconscient.

On n'y échappe pas: si le héros présenté sur scène doit lutter contre une motion refoulée, et que cette dernière se manifeste en clair en public, le spectateur normal réagira par un dégoût immédiat, une aversion massive. Le spectateur névrosé réagira, lui aussi, par une résistance, quoiqu'à un moindre degré. Dans tous les cas, donc, le dramaturge doit trouver des ruses pour ne pas déclencher ces résistances du spectateur. Freud propose trois règles que le dramaturge doit mettre en œuvre pour réussir cette opération délicate, dont Shakespeare donne un exemple très réussi dans Hamlet :

1. Le héros n'apparait pas au départ pathologique, il le devient au cours de l'action. En clinique, tous les névrosés expriment la conviction que leurs troubles sont de simples réactions aux difficultés de leur histoire.

2. La motion refoulée évoquée dans le drame doit être une motion sur laquelle s'exerce le refoulement de manière universelle chez tous les humains. Le spectateur se trouve apte au même conflit que le héros.

3. L'artiste détourne l'attention du public par un effet esthétique ; il capte son attention sur un argument, un enjeu, une intrigue qui ne sont pas des représentations refoulées; par exemple, le spectateur d'Hamlet va accompagner son héros dans sa lutte pour défendre son honneur de prince.

40 Ce faisant, « le spectateur est la proie des sentiments, au lieu de se les expliquer ${ }^{26}$ ». Il y a là libération d'affects sans ex pression des représentations refoulées qui leur correspondent, mécanisme expliquant, selon Roger Dorey, l'expérience d'inquiétante étrangeté27.

41 Le clinicien pourra trouver ces considérations inutiles pour sa pratique. Freud au contraire souligne la parenté entre la dramaturgie et la psychanalyse. Il faut épargner, contourner, éviter, ménager une part de résistance, « comme on le voit dans le travail analytique $^{28}$ ", où les sentiments que le patient ressent (on reconnaît le transfert) permettent d'atténuer les résistances.

Plus encore, Freud insiste sur la leçon que les cliniciens peuvent apprendre des dramaturges, des metteurs en scène, des acteurs. Si un névrosé nous livre un conflit tout achevé, nous ne pouvons y voir clair. Mais si nous faisons le détour nécessaire, la longue marche d'approche préalable pour connaître, pas à pas, les moments de ce conflit, ses étapes, ses passages difficiles, si nous suivons l'évolution avec l'intéressé, nous oublions qu'il s'agit d'une maladie.

Freud n'a pas découvert aisément l'explication d'Hamlet; il ne l'a pas déduite d'un raisonnement logique ; il a été le premier, après trois siècles, à la " deviner " (" erraten ${ }^{29}$ »), grâce à des analogies entre les personnages d'Hamlet et d'‘Edipe.

Par définition, le névrosé résiste à reconnaître les rejetons du refoulé comme tels; il doit sans cesse renouveler son action de répression sans "démasquer " la motion pulsionnelle. Le dramaturge qui, lui aussi, travestit les expressions pulsionnelles, 
trouve un bon public en la personne du névrosé qui méconnaît ses pulsions et leur véritable nature, qui perçoit les affects liés à cette motion sous un "nom d'emprunt ».

L'auteur dramatique présente la motion de désir refoulée sous un autre nom, qui en masque la source, qui brouille la piste. On croit Hamlet tourmenté par la soumission au désir paternel, Freud a dû lui-même deviner qu'il s'agissait d'un désir parricide.

Ce détournement de l'attention, cette diversion, la condition la plus importante selon Freud, permet un éveil direct des sentiments du spectateur sans que celui-ci prenne conscience du désir pulsionnel qui l'anime ${ }^{30}$.

Pris par l'effet esthétique, l'émotion esthétique, le spectateur va aussitôt percevoir l'intensité de son affect comme la confirmation du «faux-nom» de la motion de désir éveillée par le personnage ; ainsi la boucle est bouclée et l'émotion peut se donner libre cours, en utilisant même parfois, comme dans le mot d'esprit tendancieux, l'énergie fixée par les instances refoulantes.

Pour conclure sur ce point, rappelons que Freud ne se contente pas de chercher dans les œuvres dramatiques des illustrations de ses thèses mais qu'il admire profondément l'intelligence, l'adresse, la mesure, la maîtrise dont font preuve de grands auteurs comme Ibsen, Sophocle, Shakespeare et Schnitzler.

49 En 1900, il écrivait à propos d'Edipe-roi: «La pièce n'est pas autre chose qu'une révélation progressive et très adroitement mesurée - comparable à une psychanalyse du fait qu'€dipe lui-même est le meurtrier de Laïos, mais aussi le fils de la victime et de Jocaste ${ }^{31}$.»

50 En 1905, il reprend cela ainsi : «Ce serait la tâche du dramaturge de nous plonger dans la même maladie, ce qui se réalise au mieux quand nous suivons l'évolution avec luii ${ }^{32}$.»

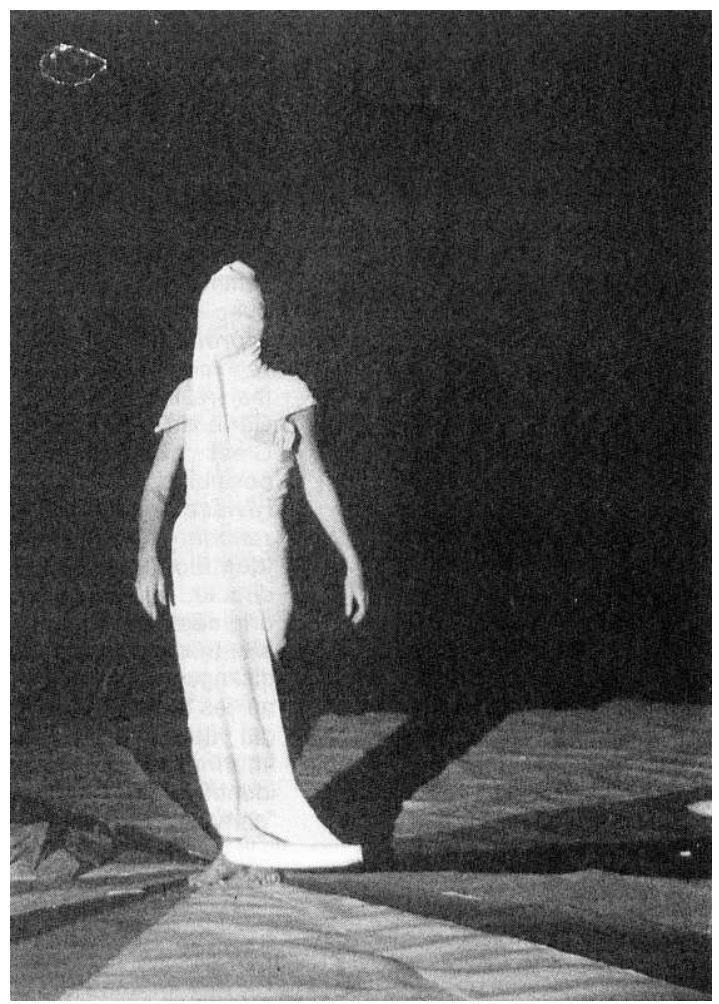

(C) Valérie Goy. 


\section{La fonction scénique}

51 J'ai proposé, en 1982, de décrire une fonction sémiotique spécifique du théâtre, la fonction scénique, en complément de la fonction poétique décrite par Roman Jakobson ${ }^{33}$. Cette proposition prenait comme point de départ un article de Freud de 1913, Le motif $d u$ choix des coffrets ${ }^{34}$. Freud vient de renverser totalement sa conception sur les rapports entre le théâtre et l'inconscient. En 1905, dans «Personnages... » il insistait sur la nécessité pour le dramaturge de détourner l'attention du spectateur du contenu pulsionnel refoulé afin d'éviter de provoquer dans le public des résistances.

Il affirme dans Les Trois Coffrets qu'au contraire, la représentation scénique permet une réduction de la déformation des représentations inconscientes, une remontée aux sources, aux situations les plus archaïques, un retour partiel à l'originel ${ }^{35}$.

Pourquoi ce renversement théorique radical, pourquoi le théâtre considéré jusqu'alors comme un exemple de déformation du contenu inconscient devient-il maintenant un moyen privilégié d'exprimer les représentations inconscientes en réduisant la déformation de celles-ci?

Je pense que l'on peut expliquer cela par la prise en compte d'une fonction spécifique de la représentation théâtrale, la fonction scénique.

Dans son article de 1913, Freud décrit et analyse deux pièces de Shakespeare. Dans Le Marchand de Venise, Portia, belle héritière, soumet ses prétendants à un choix entre trois coffrets d'or, d'argent et de plomb. Basanio réussit l'épreuve car il choisit le coffret du métal le plus humble et le plus simple, le coffret de plomb.

En référence à la symbolique du rêve, Freud interprète le choix entre trois coffrets comme le choix qu'opère un homme entre trois femmes.

Il étudie ensuite une tragédie de Shakespeare, Le Roi Lear.

Lear, de son vivant, décide de partager son royaume entre ses trois filles, proportionnellement à l'amour qu'elles lui témoigneront. On sait que la troisième, Cordélia, garde le silence; Lear partage ses biens entre les deux aînées mais Cordélia apparaîtra plus tard comme la plus digne d'être préférée.

Freud est frappé par un "facteur d'unité » (« Einheit ») entre le simple coffret de plomb, la modeste Cordélia, Cendrillon, Psyché, Aphrodite et des personnages des contes de Grimm $^{36}$. Nouvelle équation issue du symbolisme onirique : le mutisme représente la mort.

Autre facteur d'unité, dans ces divers récits, les personnages féminins sont toujours au nombre de trois, elles représentent les trois Parques, les sœurs symboles du destin, figurant les étapes de la vie humaine, la troisième représentant l'inévitable mort.

61 Mais si tel est le cas, pourquoi est-ce toujours la troisième qui est choisie?

62 Freud reconnaît ici la mise en œuvre de deux mécanismes: inversion du désir et remplacement par son contraire. En effet, on croit choisir la déesse de la beauté et de l'amour, alors qu'elle occupe la place de l'inéluctable, de l'inévitable, de l'implacable mort ${ }^{37}$ dont il est impossible de se détourner (das Unabwendbare, den Tod). Notre désir est de substituer l'amour à la mort, mais les œuvres dramatiques nous montrent que les deux pôles de cette substitution désignent une identité archaïque présente dans les divinités maternelles.

63 Ce n'est donc pas tant une déformation, un détournement de l'attention, un travestissement que le théâtre réalise. Ce qu'il présente nous paraît mystérieux 
précisément par son caractère archaïque, originel, fondamental. L'effet saisissant du spectacle s'explique par un mécanisme de réduction de la déformation. syntagmatique et paradigmatique, il attribue à chacun de ces axes des opérations spécifiques. Or sélection, opposition et substitution sont les opérations spécifiques de l'axe vertical paradigmatique soumis au principe d'équivalence. Le locuteur a le choix entre plusieurs paradigmes, distincts les uns des autres pour occuper une place unique dans la phrase ${ }^{45}$.

$$
\text { la fonction poétique), projection du principe de contiguïté de l'axe syntagmatique sur }
$$
l'axe paradigmatique du langage. 
sélectionner l'expérience préférée et substituer au choix redouté, la mort, un choix désiré, l'amour.

Tel est le coup de théâtre qui saisit Freud quand il voit Lear apparaître au fond de sa scène portant Cordélia. La fonction scénique a transposé les syntagmes successifs en les disposant pour constituer un bouquet de paradigmes entre lesquels le choix serait possible.

Or la motion critique de démenti de l'illusion nous rappelle sans cesse que cette présentation est illusoire. La représentation scénique nous fait croire que les éléments du choix peuvent à volonté être replacés dans l'ordre choisi d'une séquence à caractère réversible, comme si on pouvait remonter le temps; il n'en est rien. De même que le passage de la vie à la mort est à sens unique, sans retour, de même est inutile l'ultime prière du roi Lear cherchant à retrouver les signes de la vie dans le corps de sa fille : "Cette plume bouge, elle vit! Ah ! S'il en est ainsi, ce bonheur rachète toutes les peines que j'aie jamais souffertes ${ }^{46}$.»

Freud confirmera dans un article de 1916, « quelques types de caractère dégagés par le travail psychanalytique ${ }^{47}$ », que le héros qui nous est présenté au théâtre ne dispose que d'une liberté apparente. Dès qu'il y a conflit, les lois de l'économie poétique ou de l'économie dramaturgique imposent la reproduction forcée d'un modèle pulsionnel ou fantasmatique selon les procédés en œuvre dans les processus primaires.

Ainsi, Richard III, né avant terme avec un corps difforme, bossu et boiteux, nous touche par l'exactitude et l'amertume avec laquelle il décrit ces blessures précoces du narcissisme, l'injustice d'être spolié, blessé, handicapé dans son corps. La révolte contre ce préjudice, Gloucester l'exprime d'abord dans le renforcement de la fonction d'autoobservation décrite par Freud parmi les fonctions du Surmoi : «Eh bien, moi, dans cette molle et languissante époque de paix, je n'ai d'autre plaisir, pour passer les heures, que d'épier mon ombre au soleil et décrire ma propre difformitét ${ }^{48}$."

La déduction que Gloucester développe à partir de cette souffrance, c'est la conviction d'être une exception et d'avoir donc droit à un dédommagement qu'il s'accorde luimême, corrigeant l'injustice dont il est victime en l'infligeant aux autres.

Freud souligne deux aspects généraux du message théâtral :

- il est toujours incomplet, allusif, ambigu, proféré à voix basse, à demi-mot, retenu; le dramaturge nous oblige ainsi à compléter le message du personnage par l'écho rencontré dans nos propres souffrances et nos propres déceptions.

- d'autre part, nous retrouvons dans les traits de ce personnage un agrandissement gigantesque («Vergrösserung ») d'un aspect méconnu de notre personnalité ${ }^{49}$.

Le spectacle ravive directement une secrète connivence avec le personnage, nous invitant à comprendre sa détresse pour expliquer son application primaire d'un désir de vengeance.

Dans cet article, Freud précise nettement à quelle instance appartiennent les représentations inconscientes que le théâtre rapproche de notre conscience; elles appartiennent à ce qu'il appellera le Surmoi, avec ses fonctions d'auto-observation, les forces de la conscience morale et la fonction de l'idéal ${ }^{50}$.

Autre exemple, Shakespeare nous présente Lady Macbeth comme une femme fanatique, crispée dans une ambition effrénée mais se heurtant à l'impossibilité de maintenir à la fois une intensité absolue du désir et la durée de cette attitude. Un tel désir exacerbé ne 
peut persister (Audauern). Le théâtre le renforce jusqu'à un point culminant (Gipfelpunkt) par une contraction dans le temps puis représente son échec inévitable ${ }^{51}$. l'Acropole ${ }^{52} »$.

L'action des processus primaires domine le destin des personnages, entraînés dans les rapides d'une succession de vengeances et de crimes.

Là encore, la fonction d'auto-observation reste la plus importante, on peut sentir l'évocation d'une Lady Macbeth qui fut naguère frappée injustement dans sa féminité tendre et douce quand elle connut les joies de l'allaitement et, hypothèse personnelle, eût peut-être la douleur de perdre son enfant. Il faut avoir connu cette douleur pour se concentrer avec une telle passion sur le désir de remplacer le couple royal.

Il faudra attendre vingt ans, de 1916 à 1936, pour que Freud énonce la formule qui explique l'énigme de ces personnages tragiques «qui échouent devant le succès », dans ce texte hallucinant qu'est la lettre à Romain Rolland, «Un trouble de mémoire sur

Il écrit: ces sujets «tombent malades et même font naufrage parce qu'un de leurs désirs, doué d'une intensité exceptionnelle, a trouvé son accomplissement ». Mais l'accomplissement de ce désir intense mobilise aussitôt un interdit, une défaillance (Versagung), une frustration aussi intérieure qu'extérieure: «On ne s'accorde pas ce bonheur, le refus intérieur commande le refus extérieur. »

Ainsi fonctionnent les personnages de théâtre, pures créations de l'auteur; leur comportement extérieur est aussi soumis à leurs dispositions intérieures que leur vécu intérieur dépend des circonstances extérieures.

Cet événement, l'accomplissement d'un désir intense, nous parait trop beau pour être vrai, «car, nous le savons depuis longtemps, le destin dont on attend un mauvais traitement est la matérialisation de notre conscience, de ce sévère Surmoi qui est en nous et dans lequel s'est déposée l'instance répressive de notre enfance ${ }^{53}$ ».

Ainsi, la mort promise au héros est la figuration des interdits de son enfance.

Le personnage de théâtre est toujours pris entre deux pôles antagonistes. Parfois, cette figure d'oxymoron est représentée par un couple de personnages ; ainsi Macbeth et son épouse figurent les deux éléments d'une identité archaïque : quand elle est implacable pour décider le crime ambitieux, il frissonne de crainte; quand elle s'effondre dans la stérilité et le remords, il affronte l'adversité vaillamment ${ }^{54}$.

93 Ces personnages sont toujours composés d'un mélange d'audace et de vulnérabilité, de rage et de tendresse, de folie et de fragilité. Le personnage de théatre signifie ainsi les rapports qui unissent autour de mobiles profonds, secrets et puissants, le passé avec le présent et l'avenir, ce dont nous avons été victimes avec ce dont nous sommes coupables, ce qui concerne autrui avec ce qui nous est propre, la reproduction imposée de certaines relations avec la volonté de développer malgré tout notre liberté.

94 Car enfin, le théâtre n'est pas que tragédie. C'est aussi l'art qui nous offre la possibilité de retrouver l'expérience de ravissement, d'éblouissement de la rencontre du premier objet, cette identification précoce, immédiate et directe, ce moment de toutes premières origines où « investissement d'objet et identification ne peuvent guère être distingués l'un de l'autre ${ }^{55}$ ", ce moment où les investissements d'objet partent du Çà, quand ces choix d'objet érotique impriment au moment des premières identifications une marque qui garde un « caractère durable et général ${ }^{56}$ ». 


\section{NOTES}

1. Freud S., L'Interprétation des rêves (1900), PUF, 1967, p. 455 ; GW, III, p. 541.

2. Ibidem, p. 68 ; GW, II, p. 73.

3. Ibidem, p. 191 , note 2 .

4. Freud S., « Le rêve et son interprétation » (1901), Gallimard, 1925, p. 50 ; GW, III, p. 539.

5. Freud S., Sigmund Freud raconté par lui-même (1925), Gallimard, 1984, p. 76 ; GW, XIV, p. 71.

6. Lacan J., Écrits, Seuil, 1966, p. 511.

7. Anzieu D., L'Auto-analyse de Freud à la découverte de la psychanalyse, PUF, 1975, tome 2, p. 615.

8. Freud S., L'Interprétation des rêves, op. cit., p. 454 ; GW, III, p. 539.

9. Ibidem, p. 461.

10. Freud S., Notes à la traduction des « leçons du mardi », de J.M. Charcot, SE, vol. I, p. 137.

11. Freud » S., « Note sur l'inconscient en psychanalyse » (1912), in Métapsychologie, Gallimard, 1978, p. 179-180.

12. Freud S., L'Interprétation des rêves, op. cit., p. 58-59 ; G.W., II, p. 61.

13. Ibidem, p. 68.

14. Ibidem, p. 61.

15. Ibidem, p. 136-137.

16. Freud S., «Complément métapsychologique à la théorie du rêve » (1915), in Métaphyschologie, Gallimard (« Idées »), 1978.

17. Freud S., « Personnages psychopathiques à la scène » (1905), in Résultats, idées, problèmes, PUF, 1984, tome I, p. 123-129 ; texte allemand in Studiensausgabe, X, Bilden, Kunst und Literatur, Fischer Verlag, 1969, p. 163-168.

18. Thoret Y., "La clinique psychiatrique comme texte ", L'Évolution psychiatrique, tome 50, fasc. 3, 1985, p. 675-687.

19. Freud S., op. cit., p. 123 ; Studiensausgabe, X, p. 163.

20. Weil H., Études sur le drame antique, $3^{\mathrm{e}}$ éd., Hachette, 1897.

21. Starobinski J., « Hamlet et Freud », préface à l'ouvrage de Jones E., Hamlet et đEdipe, Gallimard, 1967, p. VII-XL.

22. Freud S., La Naissance de la psychanalyse, PUF, 1973, p. 198.

23. Freud S., «Personnages psychopathiques à la scène ", op. cit., p. 124 ; Studiensausgabe, X, p. 163.

24. Thoret Y., «En quoi le théâtre éclaire-t-il le mécanisme de l'illusion?", L'Évolution psychiatrique, tome 51, fasc. 1, 1986, p. 151-166.

25. Freud S., op. cit., p. 123 ; Studiensausgabe, p. 163.

26. Freud S., op. cit., p. 128.

27. Dorey R., "Étrangement familière, la femme », exposé à la journée d'étude du CREF le 7 juin 1986, université de Paris X.

Coulisses, 1 | Printemps 1990 
28. Freud S., op. cit., p. 128.

29. Ibidem, p. 128 ; Studiensausgabe, p. 167.

30. Ibidem, p. 128-129.

31. Freud S., L'Interprétation des rêves, op. cit., p. 228.

32. Freud S., « Personnages psychopathiques à la scène », op. cit., p. 128.

33. Thoret Y., "Étude sémiologique de la fonction scénique dans la relation thérapeutique », L'Évolution psychiatrique, tome 48, fasc. 2, 1983, p. 471-481.

34. Freud S., « Le motif du choix des coffrets », in L'Inquiétante Étrangeté et autres essais, Gallimard, 1985, p. 61-81.

35. Ibidem, p. 79.

36. Ibidem, p. 69 à 71 .

37. Ibidem, p. 76 ; GW, X, p. 33.

38. Ibidem, p. 78.

39. Ibidem, p. 81.

40. Ibidem, p. 81 ; GW, X, p. 37.

41. Ibidem, p. 81.

42. Ibidem, p. 81 ; GW, X, p. 36.

43. Ubersfeld A., Lire le théâtre, Éd. sociales, 1978.

44. Freud S., « Le motif du choix des coffrets », op. cit., p. 77 ; GW, X, p. 33.

45. Jakobson R., « Linguistique et poétique », in Essais de linguistique générale, tome I, Minuit, 1963, p. 209-248.

46. Shakespeare W., Le Roi Lear, acte V, scène 3, in Fuvres complètes, Gallimard («Pléiade »), p. 950.

47. Freud S., "Quelques types de caractère dégagés par le travail psychanalytique », in L'Inquiétante Étrangeté et autres essais, Gallimard, 1985, p. 135-171.

48. Shakespeare W., " Richard III », acte I, scène 1, in Euvres complètes, op. cit., p. 385.

49. Freud S., op. cit., p. 144-145; GW, X, p. 369.

50. Ibidem, p. 149 et 170-171.

51. Ibidem, p. 154 ; GW, X, p. 377.

52. Freud S., « Un trouble de mémoire sur l'Acropole. Lettre à Romain Rolland », in Résultats, idées problèmes, tome 2, PUF, 1985, p. 224-225; GW, XVI, p. 253.

53. Ibidem, p. 225.

54. Freud S., «Quelques types de caractère dégagés par le travail psychanalytique », op. cit., p. 157-158.

55. Freud S., « Le Moi et le Çà » (1923), in Essais de psychanalyse, Payot, 1981, p. 241.

56. Ibidem, p. 243.

57. Freud S., Correspondance, Gallimard, 1966, p. 192.

\section{AUTEUR}

\section{YVES THORET}

Yves Thoret est psychiatre au centre hospitalier de Becheville aux Mureaux. Il est maître de conférences de psychopathologie à l'Université de Paris-X Nanterre. 